\title{
Regionalized Loss of Parvalbumin Interneurons in the Cerebral Cortex of Mice with Deficits in GFR $\alpha 1$ Signaling
}

\author{
Alison J. Canty, ${ }^{1}$ Jule Dietze, ${ }^{1}$ Michael Harvey, ${ }^{2}$ Hideki Enomoto, ${ }^{3}$ Jeffrey Milbrandt, ${ }^{4}$ and Carlos F. Ibáñez ${ }^{1}$ \\ ${ }^{1}$ Department of Neuroscience, Division of Molecular Neurobiology, and ${ }^{2}$ Department of Neuroscience, Division of Brain Research, Karolinska Institute, \\ S-171 77 Stockholm, Sweden, ${ }^{3}$ Laboratory for Neuronal Differentiation and Regeneration, RIKEN Center for Developmental Biology, Kobe 650-0047, Japan, \\ and ${ }^{4}$ Department of Pathology and Immunology, Washington University School of Medicine, St. Louis, Missouri 63110
}

\begin{abstract}
Inhibitory interneurons are crucially important for cerebral cortex function and behavior. The mechanisms controlling inhibitory interneuron diversification and allocation to distinct cortical areas remain poorly understood. GDNF (glial cell line-derived neurotrophic factor) and its receptor GFR $\alpha 1$ have been implicated in the development of GABAergic precursors but, because of the early lethality of null mutants, their roles in postnatal maturation and function of cortical interneurons are unknown. "cis-only" mutant mice lack GFR $\alpha 1$ only in cells that do not express the RET signaling receptor subunit and survive to adulthood. At birth, both null mutants and cis-only mice showed a specific loss of GABAergic interneurons in rostro- and caudolateral cortical regions but not in more medial areas. Unexpectedly, the adult cortex of cis-only mice displayed a complete loss of parvalbumin (PV)-expressing GABAergic interneurons in discrete regions (PV holes) interspersed among areas of normal PV cell density. PV holes predominantly occurred in the visual and frontal cortices, and their size could be affected by neuronal activity. Consistent with deficits in cortical inhibitory activity, these mice showed enhanced cortical excitability, increased sensitivity to epileptic seizure, and increased social behavior. We propose that GFR $\alpha 1$ signaling guides the development of a subset of PV-expressing GABAergic interneurons populating discrete regions of the cerebral cortex and may thus contribute to the diversification and allocation of specific cortical interneuron subtypes.
\end{abstract}

\section{Introduction}

The cerebral cortex is comprised of both excitatory (glutamatergic) projection neurons and inhibitory (GABAergic) short-range interneurons that work together to maintain the balance between inhibition and excitation that is necessary for the correct functioning of the cortex. Cortical interneurons represent $20-30 \%$ of all neurons in the cortex and comprise a very heterogeneous group with varied morphologies, electrophysiological properties, and complement of neuropeptides, ion channels, and calcium-binding proteins (Markram et al., 2004). Abnormalities in inhibitory interneurons are apparent in several neurological conditions, including epilepsy and schizophrenia (Eyles et al., 2002; Cossart et al., 2005), and have also been proposed to be implicated in bipolar disorder and autism (Rubenstein and Merzenich, 2003; Levitt, 2005). Understanding the processes and molecules responsible for the diversification and cortical deployment of this important group of neurons is key to elucidating the

\footnotetext{
Received June 7, 2009; revised July 13, 2009; accepted July 16, 2009.

This work was funded by grants from the Swedish Foundation for Strategic Research, the Swedish Research Council (K2006-33X-10908-13-3, 315-2004-6434, and Linné program), and the European Commission (QLG3-CT2002-01000 and LSHM-CT-2004-512039 “NeuroNE”). A.J.C. was supported by a postdoctoral fellowship from NeuroNE. We thank Tibor Harkany and Per Roland for fruitful discussions and advice, Vassilis Pachnis for Lhx6 antibodies, Edgar Kramer and Rüdiger Klein for Ret mutant brains, and Niklas Lilja, Linda Thors, and Tua Finnman for help with animal care.

Correspondence should be addressed to Carlos F. Ibáñez at the above address. E-mail: carlos.ibanez@ki.se.

A. J. Canty's present address: Neuroplasticity and Disease Group, MRC Clinical Sciences Centre, Imperial College, Hammersmith Hospital Campus, London W12 0NN, UK.

DOI:10.1523/JNEUROSCI.2658-09.2009

Copyright $\odot 2009$ Society for Neuroscience $\quad$ 0270-6474/09/2910695-11\$15.00/0
}

cause of such disorders and identifying therapeutic targets for their treatment.

GABAergic interneurons are born in the embryonic subpallium in the medial, lateral, and caudal ganglionic eminences between embryonic day 12.5 (E12.5) and E15.5 in the mouse, from where they undergo a protracted period of tangential migration toward the cerebral cortex, hippocampus, and olfactory bulb (Corbin et al., 2001; Marín and Rubenstein, 2003; Levitt et al., 2004; Wonders and Anderson, 2006). Two fundamental questions that remain unsolved regarding cortical interneuron development concern where and how interneuron diversity is generated, and the mechanisms that promote and guide their migration and allocation in the cortex. Although the large morphological, neurochemical, and functional diversity of cortical GABAergic interneurons does not manifest until the postnatal maturation of these cells, part of that diversity is currently believed to arise during their generation in the ventricular zone of the embryonic ventral telencephalon. Drawing parallels to mechanisms operating in the spinal cord, recent work has identified combinatorial codes of transcription factors delineating multiple subpallial progenitor domains (Flames et al., 2007; Fogarty et al., 2007; Liodis et al., 2007; Miyoshi et al., 2007). Genetic fate-mapping and micro-transplantation studies have converged in identifying the medial ganglionic eminence (MGE) as the site in which progenitors of parvalbumin (PV)- and somatostatin (SST)-expressing cortical interneurons are generated (Butt et al., 2005; Fogarty et al., 2007). Within the MGE, recent in utero transplantation studies have suggested that $\mathrm{SST}^{+}$interneurons originate from precursors located in the dorsolateral aspect 
of the MGE, whereas more medicoventrally located domains may generate $\mathrm{PV}^{+}$interneurons (Flames et al., 2007). These advances, however, have only explained a small fraction of the diversity that is known to be present among mature cortical interneurons. In addition to transcription factors, a number of extrinsic cues can influence the development of GABAergic interneurons, including neurotrophins (Polleux et al., 2002), hepatocyte growth factor (Powell et al., 2001), semaphorins (Marín et al., 2001), and neuregulin-1 (Flames et al., 2004). However, it is not clear whether any of these factors influence the primary differentiation of MGE progenitors into GABAergic cells or their further diversification into different interneuron subtypes.

In previous work, we found that glial cell line-derived neurotrophic factor (GDNF) and its receptor GFR $\alpha 1$ promote the differentiation and migration of immature GABAergic interneurons derived from the MGE (Pozas and Ibáñez, 2005). Members of the GDNF ligand family signal by binding to GPI-anchored receptors termed GFR $\alpha 1-4$, in collaboration with signaling receptor subunits such as the RET tyrosine kinase or the p140 NCAM isoform of the neural cell adhesion molecule NCAM (Airaksinen and Saarma, 2002; Paratcha et al., 2003). Mutant mice lacking GDNF or GFR $\alpha 1$ display several deficits in subpopulations of sensory and enteric neurons, and die shortly after birth due to intestinal aganglionosis and kidney agenesis (Airaksinen and Saarma, 2002). At birth, these mutants show reduced numbers of GABAergic neurons in the cerebral cortex and hippocampus, demonstrating the importance of GDNF and GFR $\alpha 1$ for GABAergic interneuron development. Intriguingly, neither RET or NCAM are required for GABAergic interneuron migration or differentiation (Pozas and Ibáñez, 2005), suggesting that other transmembrane effectors may mediate the effects of GDNF on these cells.

The early lethality of mice lacking GDNF or GFR $\alpha 1$ prevented us from assessing the consequences of this loss for postnatal cortical development, and the organization and function of inhibitory networks in the mature cerebral cortex. In the present study, we set out to investigate these questions taking advantage of a line of mice in which the lethality caused by homozygous null mutation of Gfral has been rescued by expression of a Gfral cDNA from the Ret locus (Enomoto et al., 2004). These mice, known as "cis-only," express GFR $\alpha 1$ only in cells that express RET, such as enteric neurons and kidney, and therefore survive to adulthood. cis-only mice lack GFR $\alpha 1$ in all cells that do not express RET, including a subset of GABAergic precursors in the MGE. No gross abnormalities in either the peripheral nervous system or the CNS were found in a previous analysis of these mice (Enomoto et al., 2004).

\section{Materials and Methods}

Mice. cis-only and Gfra1 ${ }^{\text {TLZ/TLZ }}$ mice (Enomoto et al., 2004), Gad67GFP mice (Tamamaki et al., 2003), Gdnf ${ }^{+/-}$mice (Pichel et al., 1996) and $R e t^{\mathrm{flx} / \mathrm{flx}}$;Nestin-CRE (cAMP response element) mice (Kramer et al., 2006) have been previously described. cis-only and Gfral ${ }^{\text {TLZ/TLZ }}$ mice have a mixed C57/129 genetic background. Wild-type (wt) and mutant mice used in the same experiment were always derived from the same litter. We note that the line of Gfral null mice used in the present study (denoted Gfral ${ }^{\text {TLZ/TLZ) }}$ is different from the one used in our previous work by Pozas and Ibáñez (2005) (denoted $\mathrm{Gfral}^{-1-}$ ). However, both lines showed comparable reductions of cortical GABAergic neuron number at birth. Animal protocols were approved by Stockholms Norra djurförsöksetiska nämnd and in accordance with ethical guidelines of the Karolinska Institute.

Immunohistochemistry. Perfusion, fixation, and preparation of cryosections followed standard procedures. Immunohistochemistry was conducted in free floating sections according to standard protocols. Primary antibodies used were rabbit anti- $\beta$-galactosidase (Cappel, 1:4000), rabbit anti-cleaved caspase-3 (Santa Cruz Biotechnology, 1:100), rabbit anticalbindin (Swant,1:1000), goat anti-calretinin (Swant, 1:1000), rabbit anti-GFAP (DAKO, 1:800), mouse anti-NeuN (Millipore Bioscience Research Reagents, 1:200), mouse anti-PV (Swant, 1:2000), rabbit anti-PV (Swant, 1:1000), rabbit anti-Lhx6 (V. Pachnis, National Institute for Medical Research, London, UK; 1:1000), rabbit anti-somatostatin-14 (Peninsula Laboratories, 1:1000), and rabbit anti-vGAT (Synaptic Systems, 1:2000) and lectins biotin anti-Banderia simplificolia to visualize microglia (BS-1 lectin, Sigma, 1:50) and biotin anti-Solanum tuberosus to visualize blood vessels (Vector Laboratories, $20 \mathrm{mg} / \mathrm{ml}$ ).

Image analysis. Serial sections (240 and $96 \mathrm{~mm}$ apart for adult and neonate brains, respectively) were processed for each antibody to ensure complete analysis of each cell type across the cortex. The mouse brain atlas by Paxinos and Franklin (2001) was used to identify functional regions of the cortex. Quantification of calbindin $(\mathrm{Cb})$ cell profiles in neonatal cortex was performed in three sagittal sections from each hemisphere (Fig. 1A). Cells were counted in rostrolateral, caudolateral, rostromedial, and caudomedial regions of 5 hemispheres per genotype from the pial surface to the underlying white matter. PV holes in the adult cis-only cortex were defined as spanning at least two consecutive sections $240 \mathrm{~mm}$ apart on the mediolateral axis and $150 \mathrm{~mm}$ in length on the rostro-caudal axis. PV holes were mapped in coronal and sagittal sections and then manually transferred onto the flattened cortical map from the Paxinos atlas (Paxinos and Franklin, 2001). Quantification of NeuN cells was performed in cortical layers $2 / 3$ of PV holes ( 5 mice, 8 holes, 44 fields) and equivalent areas of wild-type cortex ( 6 hemispheres, 125 fields) from $20 \times$ confocal images using automations made in OpenLab software (Improvision). Quantification of Gad67-GFP and Lhx6 cells was performed on sagittal sections using a grid of $0.04 \mathrm{~mm}^{2}$ (Gad67-GFP) or $0.017 \mathrm{~mm}^{2}$ (Lhx6) quadrants. Due to high variability between different animals, cell counts inside PV holes were normalized to counts obtained from an area of equivalent size surrounding each hole, and expressed as percentage cell loss. Identical areas in size and location were selected in wild-type brains (pseudoholes) and quantified for comparison. Quantification of vGAT intensity was performed in sections from PV holes and equivalent areas in wild-type controls that were processed at the same time and imaged by confocal microscopy under identical conditions. vGAT pixel intensity was measured in 6 quadrants from 2 separate images within each hole (or pseudohole) and individually adjusted for background using average pixel intensities from the underlying immunonegative corpus callosum in the same section. Unless otherwise indicated, Student's $t$ test was used for statistical analysis.

Optic nerve transection. Within 48 h of birth, pups ( $n=12$ cis-only, $n=$ 4 wild type) were anesthetized by hypothermia for $3-4 \mathrm{~min}$. A vertical cut was made overlying each eye, the optic nerve severed using fine dissection scissors, and the eye removed. The overlying skin was then rejoined, and pups were warmed on a heat pad before being returned to the home cage. Pups were given two doses of buprenorphine (Temgesic, ScheringPlough, $0.2 \mathrm{mg} / \mathrm{ml}$, s.c.) both before $(-1 \mathrm{~h})$ and after $(+12 \mathrm{~h})$ surgery to minimize pain and discomfort. This protocol resulted in $90 \%$ survival of pups. Pups remained with their mothers in the home cage until postnatal day 35 (P35), when they were genotyped and perfused. Mice raised in normal conditions ( $N=8$ per genotype) were used as controls.

Electroencephalography. Adult cis-only $(n=5)$ and wild-type $(n=5)$ mice of both sexes were deeply anesthetized with ketamine-xylazine (100 and $20 \mathrm{mg} / \mathrm{kg}$, i.p.), placed in a stereotaxic frame, and a large craniotomy was made over the left cerebral hemisphere. Local field potential recordings were made with bipolar, tungsten electrodes guided to the V2 region of the cortex using stereotaxic coordinates (Paxinos and Franklin, 2001). Signals were amplified, digitized, and acquired using standard procedures.

Seizure induction. Adult cis-only $(n=16)$ and wild-type $(n=28)$ mice aged between 2 and 7 months, of both sexes were injected with pentylenetetrazole (PTZ) (Sigma, $40 \mathrm{mg} / \mathrm{kg}$ in $0.9 \%$ saline, i.p.). Immediately after injection, each mouse was placed in a large Plexiglas cage and its behavior recorded with a Pentax digital video camera for $60 \mathrm{~min}$. Seizure severity was recorded on a scale of $0-5$ according to stages described by Lau et al. (2000). Statistical analysis was performed by Mann-Whitney 

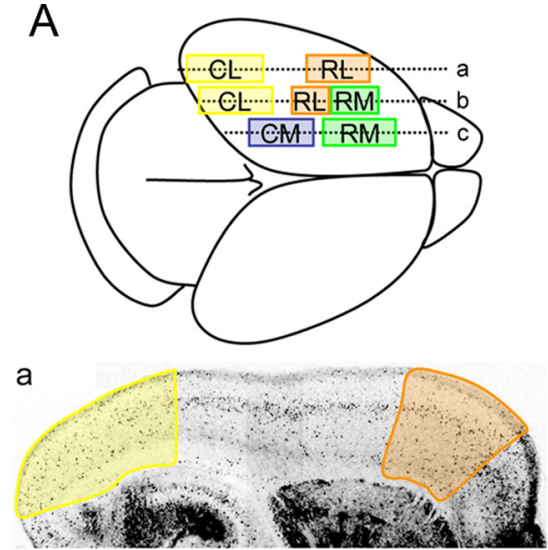

b
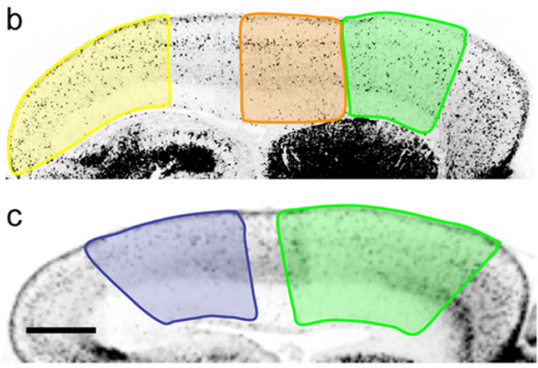

B
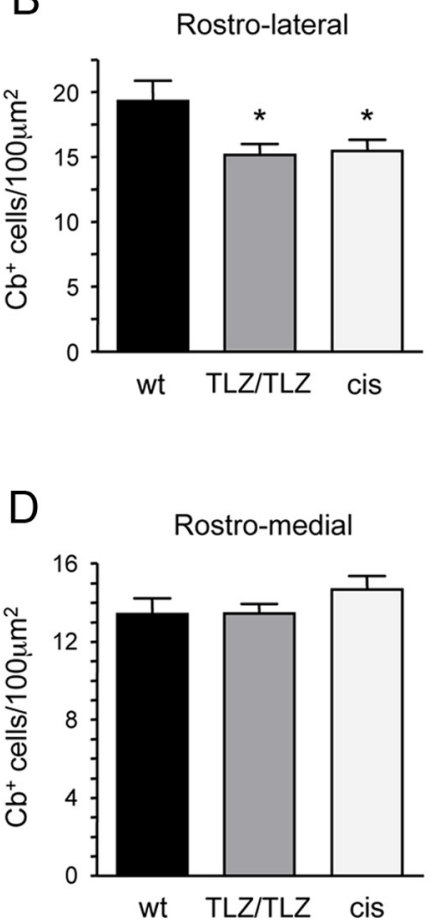

C

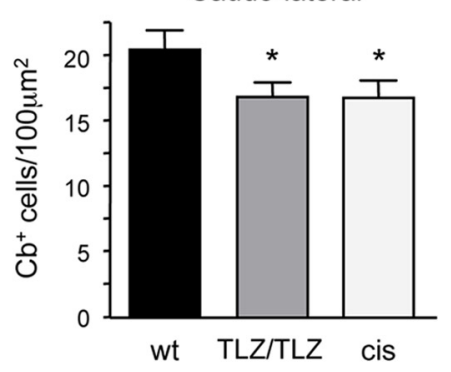

$E$

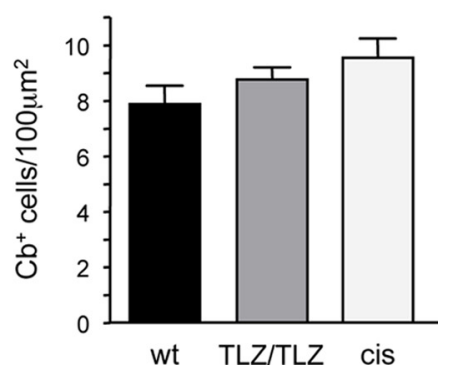

Figure 1. Region-specific loss of cortical GABAergic interneurons in newborn Gfra $1^{\mathrm{TLZ} / \mathrm{TLZ}}$ and cis-only mice. $A$, Scheme outlining the cortical regions used for quantification of Cb cells in wild-type and mutant newborn mice. Planes of sagittal sections are indicated by lines labeled $\boldsymbol{a}-\boldsymbol{c}$ and shown below with cortical areas color-coded: rostrolateral (RL, orange), caudolateral ( $\mathrm{CL}$, yellow), rostromedial (RM, green), and caudomedial (CM, blue). Scale bar, $500 \mu \mathrm{m}$. B-E, Calbindin cell counts in the indicated cortical areas of wt, Gfra 1 null mutants (TLZ/TLZ), and cis-only (cis) newborn mice. Results are expressed as average \pm SD ( $n=5$ hemispheres per genotype). ${ }^{*} p<0.05$.

nonparametric analysis (severity), or Student's $t$ test (latency). At the conclusion of behavioral observation, cis-only mice were perfused for retrospective histological analysis.

Behavioral analysis. SHIRPA and elevated plus maze followed previously established protocols (Rogers et al., 1997). For social interaction tests, adult cis-only $(n=16)$ and wild-type $(n=14)$ male mice were placed in a large arena for $11 \mathrm{~min}$, the first minute with an opaque dividing wall in the center, for two consecutive days (habituation phase). On the third day, a second previously unknown mouse of the same age, weight, and genotype, that had been subjected to identical preconditioning, was introduced in the other half of the cage (experimental phase). The behavior of each mouse was assessed on days 1 and 3 by means of subsequent analysis of video footage using a Pentax digital video camera. Behavior was categorized into 11 broad categories-i.e., body care, digging, nonsocial exploration, explore from a distance, social investigation, threat, attack, avoidance/flee, defensive/submissive, sexual, and immobility-based on existing paradigms (Estelles et al., 2007). The latency to each event, number of events, and time spent within $7 \mathrm{~cm}$ (less than one body-length) was recorded.

\section{Results}

Region-specific loss of cortical GABAergic interneurons in newborn Gfral $1^{\text {TLZ/TLZ }}$ and cis-only mice

In view of earlier findings indicating reduced numbers of cortical GABAergic neurons in newborn mice lacking GFR $\alpha 1$ (Pozas and Ibáñez, 2005), we investigated whether this deficit was also present in newborn cis-only mice (Gfral ${ }^{\text {TLZ/TLZ }} ;$ Ret $^{\text {Gfral/+ }}$ ). Although our previous study was limited to the prospective somatosensory cortex, we have extended this analysis to other cortical areas in newborn Gfral ${ }^{\text {TLZ/TLZ }}$ (null) and cis-only mice (Fig. $1 A)$. We found identical reductions in the number of cells expressing $\mathrm{Cb}^{+}$, a general marker of immature GABAergic precursors, in rostrolateral and caudolateral areas (corresponding to prospective frontal/somatosensory and visual cortices, respec- tively) in both Gfral ${ }^{\text {TLZ/TLZ }}$ and cis-only mice (Fig. $1 B, C$ ). This indicated that cis-only mice recapitulate the deficits in GABAergic neurons observed in the cortex of null mutants at birth. Unexpectedly, neither Gfral ${ }^{\text {TLZ/TLZ }}$ or cis-only mice showed any loss of $\mathrm{Cb}^{+}$cells in more rostromedial and caudomedial areas, corresponding to prospective motor and retrosplenial cortices (Fig. $1 D, E)$. This indicated that not all regions of the cerebral cortex are equally affected by the loss of GFR $\alpha 1$ expression.

\section{Parvalbumin holes in the cerebral cortex of adult cis-only mice}

During the first postnatal weeks, GABAergic neurons cease their migration and begin to mature functionally. Several different classification systems for mature interneurons have been proposed, based on their morphology, electrophysiological properties, or expression of ion channels, calcium-binding proteins, and neuropeptides (Markram et al., 2004). We used a combination of $\mathrm{PV}, \mathrm{Cb}$, and calretinin markers, labeling largely nonoverlapping subpopulations of mature interneurons, to assess the distribution of GABAergic neurons in the cortex of adult cis-only mice. It should be noted that, although widely expressed across different classes of GABAergic precursors during early stages of development, $\mathrm{Cb}$ labels a restricted subset of mature interneurons in the adult cortex (Alcantara et al., 1996). In the wild-type cortex, PV neurons normally comprise $\sim 40 \%$ of all interneurons, and their large cell bodies are distributed throughout layers 2-6 across the entire cortex (Fig. 2A,B). Unexpectedly, the cortex of cis-only mice displayed areas completely devoid of PV neurons (herein referred to as PV holes) interspersed among regions of normal PV cell density (Fig. $2 A^{\prime}, B^{\prime}$ ). PV holes were present in the majority $(82 \%)$ of cis-only brains analyzed, and either encompassed only the upper layers of the cortex (e.g., Fig. 2E,F), or the full depth of 

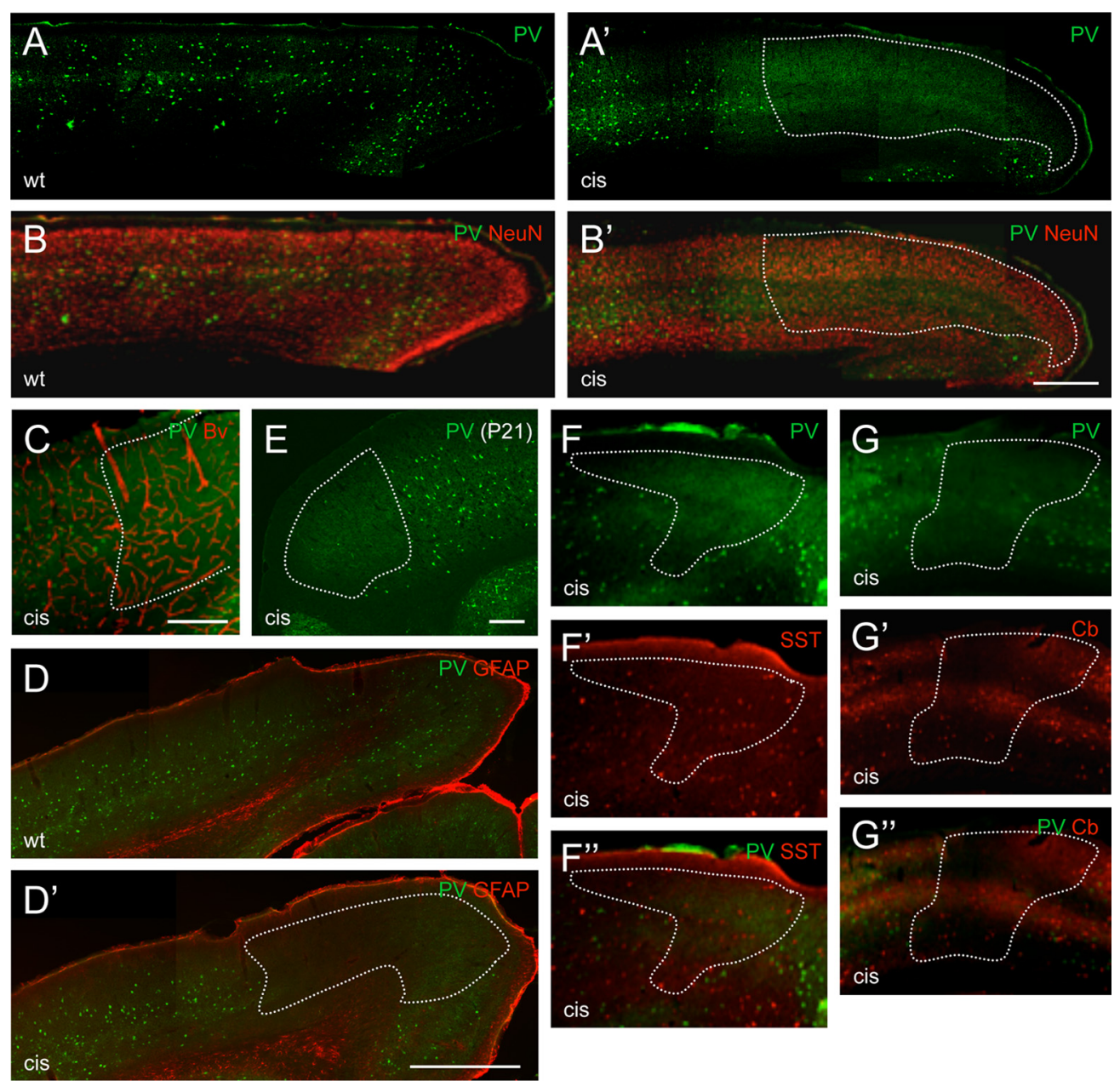

Figure 2. Parvalbumin holes in the cerebral cortex of adult cis-only mice. $\boldsymbol{A}, \boldsymbol{B}, \mathrm{PV}$ and NeuN immunohistochemistry of sagittal sections of wild-type $(\boldsymbol{A}, \boldsymbol{B})$ and cis-only $\left(\boldsymbol{A}^{\prime}, \boldsymbol{B}^{\prime}\right)$ adult cortex. PV holes are delineated by white dotted lines. Scale bar (in $\left.\boldsymbol{B}^{\prime}\right) \boldsymbol{A}, \boldsymbol{B}, \boldsymbol{A}^{\prime}, \boldsymbol{B}^{\prime}, 500 \mu \mathrm{m}$. $C$, PV and blood vessel (Bv) immunohistochemistry in PV hole and adjacent areas of a sagittal section through the adult cis-only cortex. $\boldsymbol{D}$, PV and GFAP immunohistochemistry of sagittal sections of wild-type $(\boldsymbol{D})$ and cis-only $\left(\boldsymbol{D}^{\prime}\right)$ adult cortex. Scale bar, $500 \mu \mathrm{m}$. $\boldsymbol{E}, \mathrm{PV}$ immunohistochemistry of a PV hole and adjacent areas in a sagittal section through the cortex of P21 cis-only mice. $F$, PV and SST immunohistochemistry of a PV hole and adjacent areas in a sagittal section through the adult cis-only cortex. $G$, PV and Cb immunohistochemistry of a PV hole and adjacent areas in a sagittal section through the adult cis-only cortex. Scale bars (in $C, E$ ) C, E, F, G, $200 \mu \mathrm{m}$.

the cortical plate (e.g., Fig. $\left.2 A^{\prime}, C, D, G\right)$. No PV holes were observed in the cortex of wild-type or Ret $t^{G \text { fral / }+}$ mice. Smaller, isolated PV holes, which never reached the full depth of the cortex, were observed in a fraction $(<40 \%)$ of $G$ fral $1^{\text {TLZ/+ }}$ heterozygous mutants, irrespectively of the presence of the Ret ${ }^{G \text { fral }}$ allele. Also $40 \%$ of $G d n f^{+/-}$heterozygous mutants analyzed displayed cortical PV holes (data not shown). No PV holes were observed in brains of adult Ret ${ }^{\mathrm{flx} / \mathrm{flx}} ;$ Nestin-CRE conditional mutants lacking RET in the CNS (data not shown).

No alteration in the overall neuronal structure and layering of the cortex was observed in areas lacking PV neurons as assessed with the pan-neuronal marker NeuN (Fig. $2 B, B^{\prime}$ ). The morphology of blood vessels was also normal (Fig. $2 C$, data not shown), indicating that loss of PV immunoreactivity was not a perfusion or fixation artifact. In addition, no glial reaction was observed in PV holes using markers of astrocytes (Fig. 2D, $D^{\prime}$ ), oligodendrocytes and microglia (data not shown). Neither was any increase in cell death detected using antibodies against the activated form of caspase-3 at all stages examined (E12.5-E17.5, P0, P7, P14, P21, and adult) in the cis-only cortex (data not shown).

PV expression normally develops during the second and third postnatal weeks, and PV holes could be detected as early as P21 (Fig. 2E), the earliest time by which the distribution of PV throughout the cortex begins to stabilize. It was also clear that, among the GABAergic subpopulations examined, PV interneurons were those mostly affected. No abnormalities could be detected in Calretinin-expressing interneurons, which are concentrated in the deeper layers of the cortex (data not shown). SST-expressing neurons, the other major interneuron subpopulation derived from the MGE, were also normally distributed throughout the cis-only cortex, including within PV holes (13 holes analyzed) (Fig. $2 F, F^{\prime}, F^{\prime \prime}$ ). Overall, the distribution $\mathrm{Cb}^{+}$interneurons was also normal in the cis-only cortex, including in the majority of PV holes (Fig. $2 G, G^{\prime}, G^{\prime \prime}$ ), although a fraction of these (14 of 54 analyzed) also lacked $\mathrm{Cb}$-expressing cells in a smaller volume $(62.31 \pm 5.29 \%)$ in the center of the hole (supplemental Fig. S1, available at www.jneurosci.org as supplemental material).

Loss of PV interneurons in the cis-only cortex was accompanied by a proportional reduction in GABAergic markers that was restricted to the areas lacking PV expression. A GFP gene inserted in the Gad67 locus marks the majority of GABAergic neurons in the adult cortex (Tamamaki et al., 2003), and we found a reduction of $20.4 \pm 4.6 \%$ in the number of Gad67-GFP ${ }^{+}$cells in PV holes of cis-only mice carrying this allele (Fig. $3 A, B$; supplemental Fig. $\mathrm{S} 3 A$, available at www.jneurosci.org as supplemental material). Similarly, Lhx6 specifically marks MGE-derived GABAergic neu- 
A
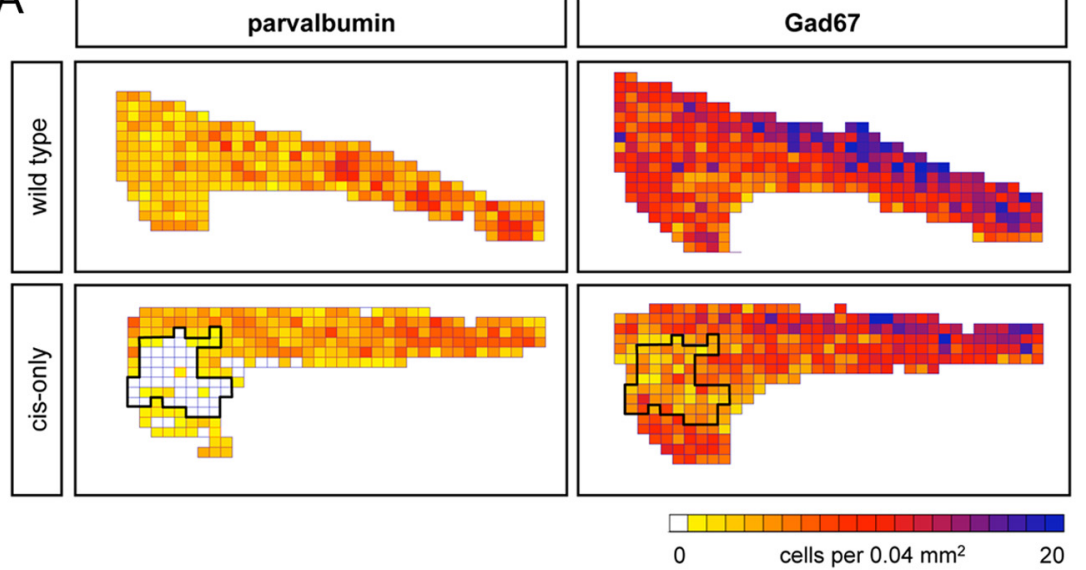

C

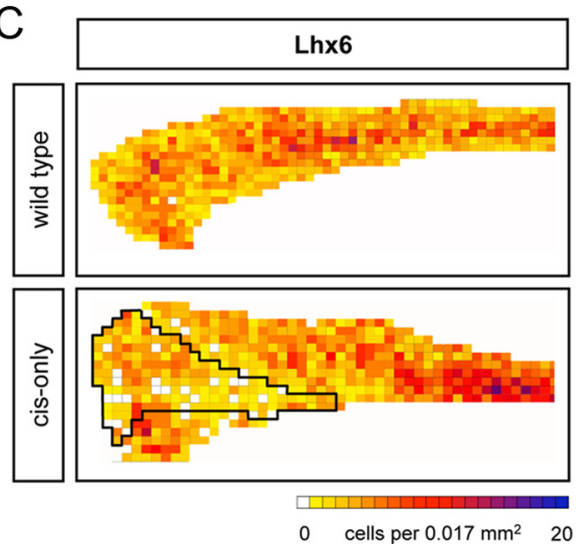

$B$

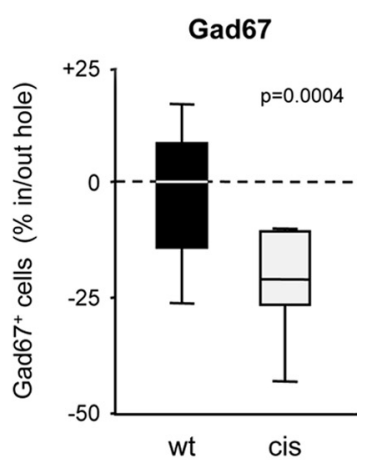

Figure 3. Neuronal loss in PV holes of cis-only mice. A, Quantitative analysis of PV and Gad67 cell density in sagittal sections of adult wild-type and cis-only cortex. Cell density in each quadrant is color-coded as per the scale shown, from 1 to 20 cells per $0.04 \mathrm{~mm}^{2}$. A PV hole is outlined in the cis-only section. $B$, Quantification of Gad67 cell number in PV holes of the adult cis-only cortex and equivalent areas of wild-type cortex. $n=6$ holes, cis-only; $n=30$, wt. C, Quantitative analysis of Lhx6 cell density in sagittal sections of wild-type and cis-only adult cortex. Cell density in each quadrant is color-coded, from 1 to 20 cells per $0.0017 \mathrm{~mm}^{2}$. A PV hole is outlined in the cis-only section. $D$, Quantification of Lhx6 cell number in PV holes of the adult cis-only cortex and equivalent areas of wild-type cortex. $n=7$ holes, cis-only; $n=6$, wt.

rons in the adult cortex (Lavdas et al., 1999), and we observed a decline of $35.7 \pm 9.08 \%$ in $\mathrm{LhX}^{+}$cell density in PV holes of cis-only mice by immunofluorescence (Fig. 3C,D; supplemental Fig. S3B, available at www.jneurosci.org as supplemental material). These values are in good agreement with the proportion of $\mathrm{PV}$ neurons among cortical interneurons and suggest a loss of PV cells, rather than loss of PV expression, in PV holes of the cis-only cortex. We also note that, outside PV holes, PV cell density in the cis-only cortex was not increased compared with wild-type cortex (Fig. $3 A$ ), arguing against the possibility that cortical PV interneurons were simply redistributed in the mutant. The analysis of GABAergic markers did not rule out the possibility that cells that failed to become GABAergic might still be present in place of PV neurons in the affected areas. To address this possibility, we counted the total number of neurons using the panneuronal NeuN marker in PV holes of cis-only mice and equivalent cortical areas of wild-type brains. We found a significant reduction of $8.4 \pm 2.46 \%(n=44$ fields, cis-only; $n=$ 125 fields, wt; $p=0.01$ ) in the total number of neurons in PV holes of the cis-only cortex, a value that is in good agreement with the proportion of PV neurons present in the cerebral cortex (Tamamaki et al., 2003). We conclude from this analysis that PV holes represent cortical areas of the cis-only brain that lack PV interneurons.

\section{Preferential distribution of PV holes in the visual and} frontal cortices

PV holes were variable in size (average volume $0.30 \pm 0.44 \mathrm{~mm}^{3}$ ). Their number and location also varied between different mutant brains, although during our initial analysis we noted a tendency for them to predominantly appear in the caudal- and frontalmost regions of the cortex. To obtain a more accurate record of their distribution, we systematically mapped the location of PV holes across serial sagittal sections in 36 hemispheres of adult cis-only brains according to their rostrocaudal and mediolateral position. These coordinates were transferred onto a flattened map of the mouse cortex (Paxinos and Franklin, 2001) (Fig. 4A), and layered upon each other to generate a heat map of PV holes in the adult cis-only cortex with a graded color scale showing the degree of overlap among holes in different hemispheres (Fig. $4 B$ ). This analysis revealed that the two cortical areas that were mostly affected by PV holes were the caudomedial visual cortex (particularly V2M and V1) and the frontal cortex, particularly the insula and frontal association cortices adjacent to the somatosensory cortex (Fig. $4 B$ ). Other areas, such as the motor, retrosplenial and auditory cortices, were either not at all or much less frequently affected (Fig. $4 \mathrm{~B}$ ). We note that this was in good correspondence with the regions showing reduced numbers of GABAergic neurons in the newborn cis-only cortex (Fig. 1), suggesting that the 
A
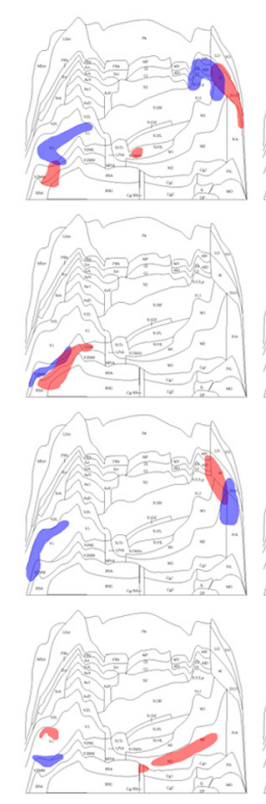
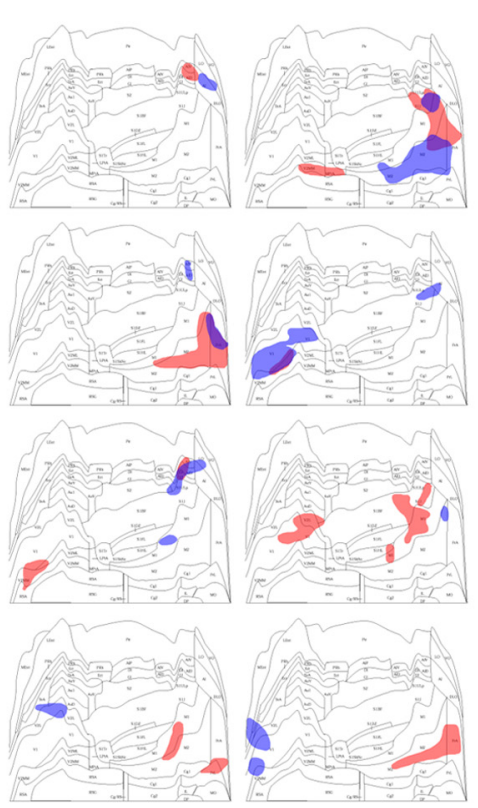

B
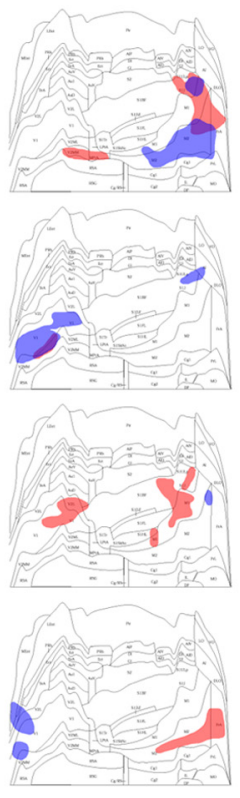

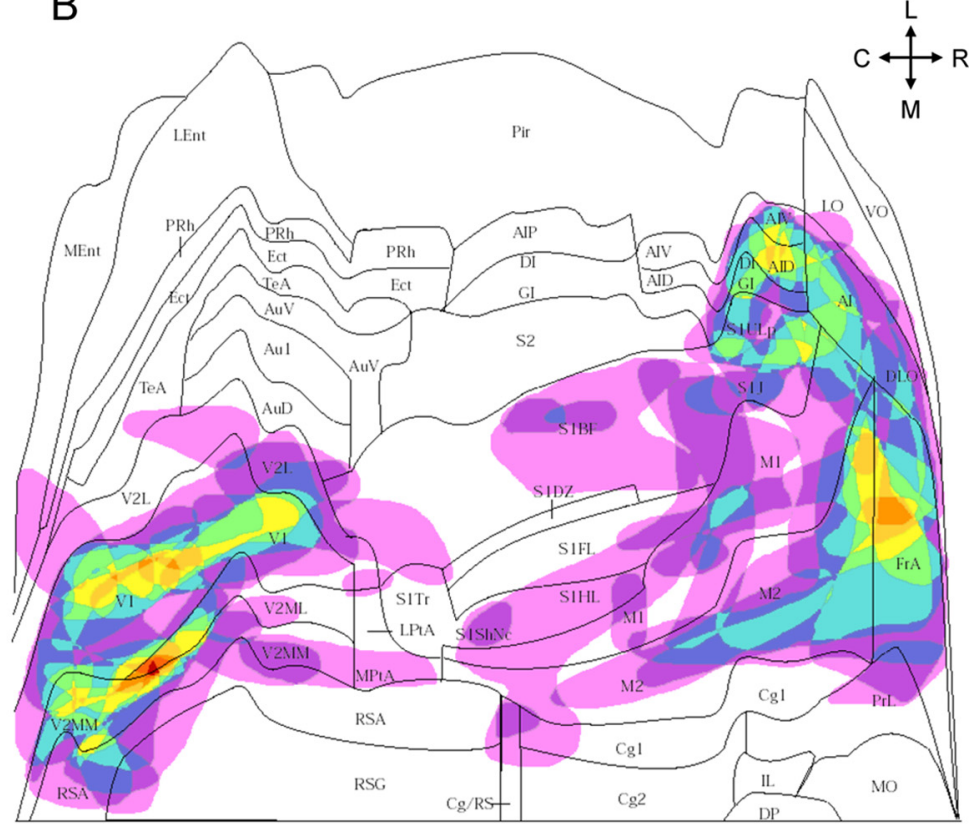

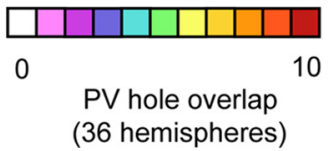

(36 hemispheres)

Figure 4. Preferential distribution of PV holes in the visual and frontal cortices. A, Distribution of PV holes on a flattened map of the mouse cortex. Shown are 12 cis-only brains with PV holes in the left (red) and right (blue) hemispheres superimposed. The midline is at the bottom; caudal is to the left. The first six brains are examples of strong symmetry, the next four have partial symmetry, and the last two show no symmetry between left and right hemispheres. $\boldsymbol{B}$, Heat map of 36 cis-only hemispheres superimposed on each other with overlap between PV holes color coded as per scale bar shown below. Two hot spots can be discerned in the visual and frontal cortices where 10 PV holes overlap. C, caudal; L, lateral; R, rostral; M, medial.

two deficits may be ontogenetically related. This was supported by our observation of a relatively high prevalence $(83.33 \%)$ of PV holes showing symmetrical distribution in the two hemispheres of the same brain (Fig. 4A), arguing against a purely stochastic process. Finally, we note that the distribution or size of PV holes was not different between aged (>12 months) and young (5 weeks) cis-only animals (supplemental Fig. S2, available at www. jneurosci.org as supplemental material).

\section{Neuronal activity affects the size but not the distribution of PV holes}

Although there was a clear bias in the location and distribution of PV holes, their precise borders were never identical among different hemispheres. In an attempt to identify additional processes that may act in concert with developmental effects to affect the position and size of individual PV holes, we examined the role of neuronal activity. In the visual cortex, the onset of PV expression is regulated by activity, principally coming in the way of light via the retina, and the appearance of PV defines the end of the critical period of plasticity. Prolongation of the critical period by dark rearing of neonatal mice, has been shown to decrease PV expression in the visual cortex (Tropea et al., 2006). To determine whether the position or size of PV holes in visual cortex can be influenced by activity, we bilaterally transected the optic nerves of neonatal mice ( $\mathrm{P} 0-\mathrm{P} 2)$ to remove both spontaneous and lightdependent retinal activity reaching the visual cortex. We then examined the distribution of PV interneurons and the incidence of PV holes in the visual cortex 5 weeks later (P35). Optic nerve transection (ONT) decreased the density of PV interneurons in primary visual cortex of both wild-type (20.9\% reduction, $p=$
0.01 ) and cis-only ( $12.3 \%$ reduction, $p=0.02)$ mice (Fig. $5 A)$. PV holes were observed in the visual cortex of both control and ONT cis-only mice at P35 (Fig. 5B). The distribution of PV holes across the cortex was not affected by ONT (Fig. 5C), and the incidence of $\mathrm{PV}$ holes in the visual cortex of control versus ONT cis-only mice was comparable ( $26 \%$ vs $30 \%$, respectively). However, we observed a threefold increase in the size of PV holes in the visual cortex of cis-only mice after ONT compared with control (Fig. 5D). ONT did not affect PV hole size in other cortical areas (Fig. $5 D$ ), indicating that this effect was specifically caused by deprivation of activity in the visual cortex. These data indicate that neuronal activity can contribute to the formation of PV holes, and suggest that differences in activity may, at least in part, account for the variable size of PV holes observed among individual cisonly mice.

\section{Decreased inhibitory activity in the cerebral cortex of cis-only mice}

PV cortical interneurons comprise fast spiking basket and chandelier cells, two very important classes of inhibitory interneurons that restrict the activity of excitatory pyramidal neurons through powerful perisomatic and axo-axonic inhibitory synapses (Markram et al., 2004). In agreement with the loss of PV interneurons, we observed a significant reduction in inhibitory innervation in PV holes of cis-only mice as assessed by quantitative immunohistochemical analysis of the vesicular GABA transporter (vGAT), a marker of inhibitory synapses (Fig. 6A; pixel intensity $2.1 \pm 0.2$ cis-only vs $4.7 \pm 0.3$ wild type; $n=24$, i.e., 6 measurements from 4 holes). This decline in inhibitory innervation was reflected in electroencephalographic (EEG) recordings 


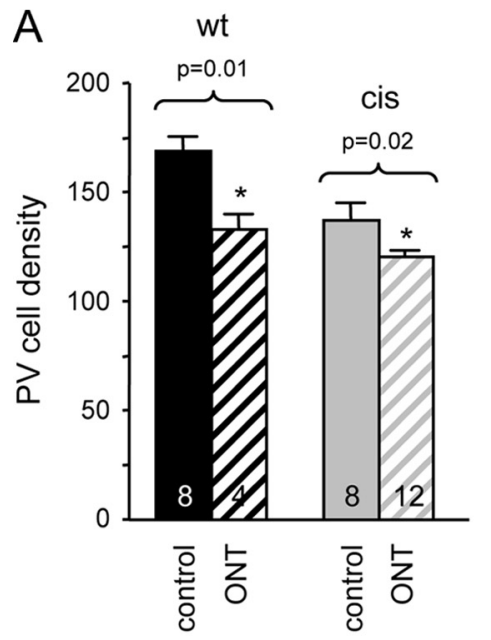

B

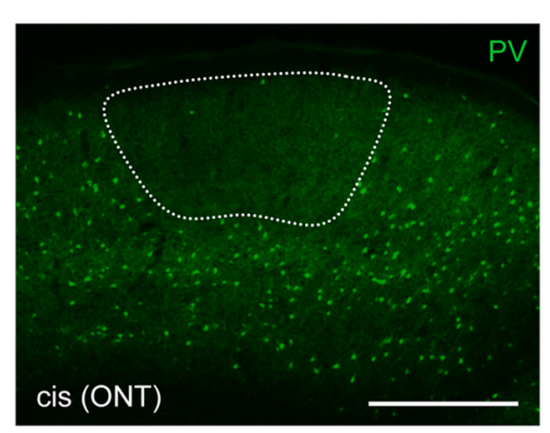

C
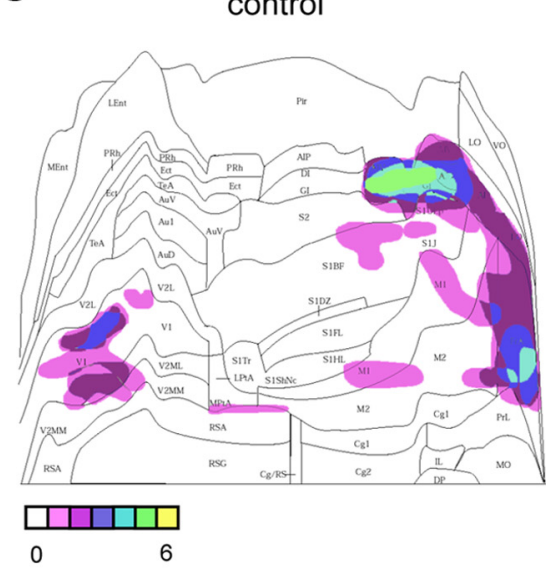

D

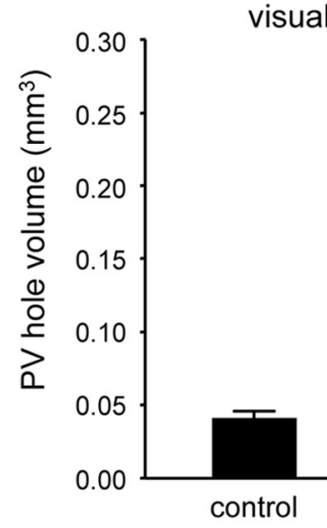

\begin{abstract}
PV holes in
visual ctx
\end{abstract}

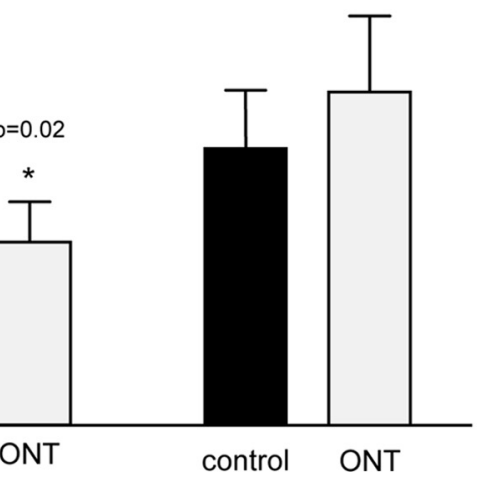

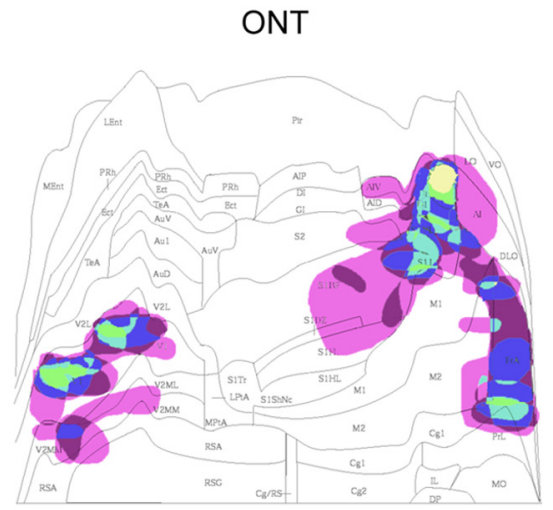

Figure 5. Neuronal activity affects the size but not the distribution of PV holes. $A, P V$ cell density in the cortex of control and ONT wild-type and cis-only mice at P35. Results are expressed as average \pm SD (number of hemispheres used is indicated). $\boldsymbol{B}$, Example of a PV hole in the visual cortex of a P35 cis-only mouse after ONT. Scale bar, $500 \mu \mathrm{m}$. C, Distribution of PV holes in P35 cis-only cortex of control ( 8 hemispheres superimposed) and ONT (13 hemispheres superimposed) mice. D, PV hole volume in visual cortex of control ( $n=5$ holes) and 0NT ( $n=13$ holes) cis-only mice. PV hole volume outside the visual cortex of control ( $n=14$ holes) and ONT ( $n=28$ holes) cis-only mice.

collected from cis-only mice under ketamine-xylazine anesthesia. In wild-type mice, recordings from a single bipolar electrode inserted into the visual cortex showed the characteristic slow oscillatory activity $(0.8-0.9 \mathrm{~Hz})$ that has previously been described in cats and humans during normal slow wave sleep (Steriade et al., 1993) (Fig. 6B). In cis-only mice, this oscillatory activity was regularly interrupted by sharp, short-lasting (30-100 ms), negative potentials ( $n=10$ electrodes, 5 mice) (Fig. $6 B$ ). These events only occurred during the depolarizing phase of the slow oscillation and are indicative of a state of enhanced cortical excitability (Lau et al., 2000). Retrospective PV immunohistochemistry was performed to confirm that PV holes were present at, or near, electrode insertion sites (data not shown). Together, these results indicate functional deficits in inhibitory cortical activity in cisonly mutants. In agreement with these observations, cis-only mice displayed a lower threshold for generation of seizures by a sublethal dose of the GABA antagonist pentylenetetrazole (PTZ). Compared with wild-type controls, cis-only mice displayed a marked increase in seizure severity to the same dose of PTZ, with 11 of 16 mice $(69 \%)$ reaching a stage 5 seizure that was always lethal in these mice (Fig. 6C). (The only wild-type mouse that reached stage 5 survived the seizure.) In addition, the latency to onset of seizure was significantly lower in cis-only mutants compared with wild-type mice (48s cis-only vs $82 \mathrm{~s}$ wild type; $p=$
$0.05 ; n=27$, wt; $n=16$, cis-only), which is also an indication of increased cortical excitability.

\section{cis-only mice show increased social behavior}

Human neurological conditions associated with abnormalities in cortical interneurons, such as schizophrenia, bipolar disorder and autistic spectrum disorders, present characteristic behavioral tendencies affecting anxiety state and social behavior. We subjected cis-only mice $(n=37)$ and their wild-type littermates $(n=23)$ to a primary SHIRPA screen to obtain a behavioral observation profile of the mutants (Rogers et al., 1997). The most prominent difference between wild type and mutants was found in the touch escape paradigm, in which cis-only males showed a heightened response $(p=0.05)$. Such responses can sometimes be associated with increased anxiety. However, no significant differences could be found in the elevated plus maze, a standard behavioral test to detect changes in anxiety, although there was a tendency for cis-only mice to spend more time in the closed arms of the maze (data not shown). Because our animals originate from a mixed C57/129 genetic background, it is possible that this difference becomes statistically significant in mice that are genetically homogenous.

To investigate the possibility of altered social behavior, we performed a social interaction test in which two animals of the 
same age, sex, and genotype that have not been in previous contact are placed together in a large open field. Before the test, each mouse had been habituated to the open field during $10 \mathrm{~min}$ for two consecutive days. Both wild-type and cis-only mice showed identical habituation profiles to the new environment in the sessions before the test, as assessed by the frequency of body care, digging, and rearing events (data not shown). On the third day, two mice were put together and the time spent in social interaction (within less than one-body length from each other) was measured during a period of $10 \mathrm{~min}$. We found that cis-only mice spent significantly more time in close contact with each other compared with wild-type littermates, as assessed by the time spent within one body-length of each other during a $10 \mathrm{~min}$ period (total interaction time 207 s cis-only vs 155 s wild type; $p=0.04$; $n=7$ pairs, wt; $n=8$ pairs, cis-only). Moreover, and in contrast to wild-type mice, they showed no habituation to the presence of an unfamiliar mouse during the testing period (Fig. $7 B$ ). We conclude from this analysis that cis-only mice display an abnormal increase in social behavior.

\section{Discussion}

In this study, we investigated the importance of the GDNF receptor GFR $\alpha 1$ for the diversification and allocation of cortical GABAergic interneurons. To this end, we used a line of mutant mice with a selective deficit in GFR $\alpha 1$ expression restricted to cells that lack the RET signaling receptor subunit. Unlike null mutants, cisonly mice survive to adulthood, likely because of restoration of GFR $\alpha 1$ expression in kidney and enteric neurons (Enomoto et al., 2004). This allowed us to investigate the fate and function of GABAergic interneurons in the postnatal and adult cortex of these mice. In newborn mutant animals, both null and cis-only, we found a specific loss of GABAergic neurons in rostrolateral and caudal areas of the cortex but not in other cortical regions. Two striking observations were made in adult cisonly mutants. First, we found a loss of cortical GABAergic interneurons that was mostly restricted to PV-expressing cells, sparing calretinin-, somatostatin-, and most calbindin-expressing interneurons. Second, the extent of this loss was quite unusual, in that it was restricted to discrete patches, which we have called PV holes, interspersed among an otherwise normallooking cortex. PV holes were most frequent in the same areas affected in newborn ani-
A
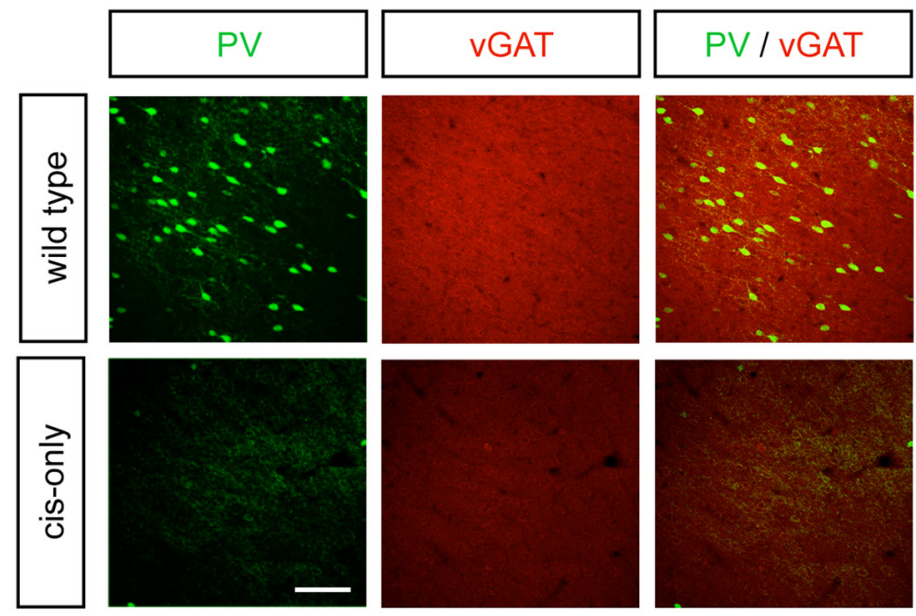

B

cis

wt
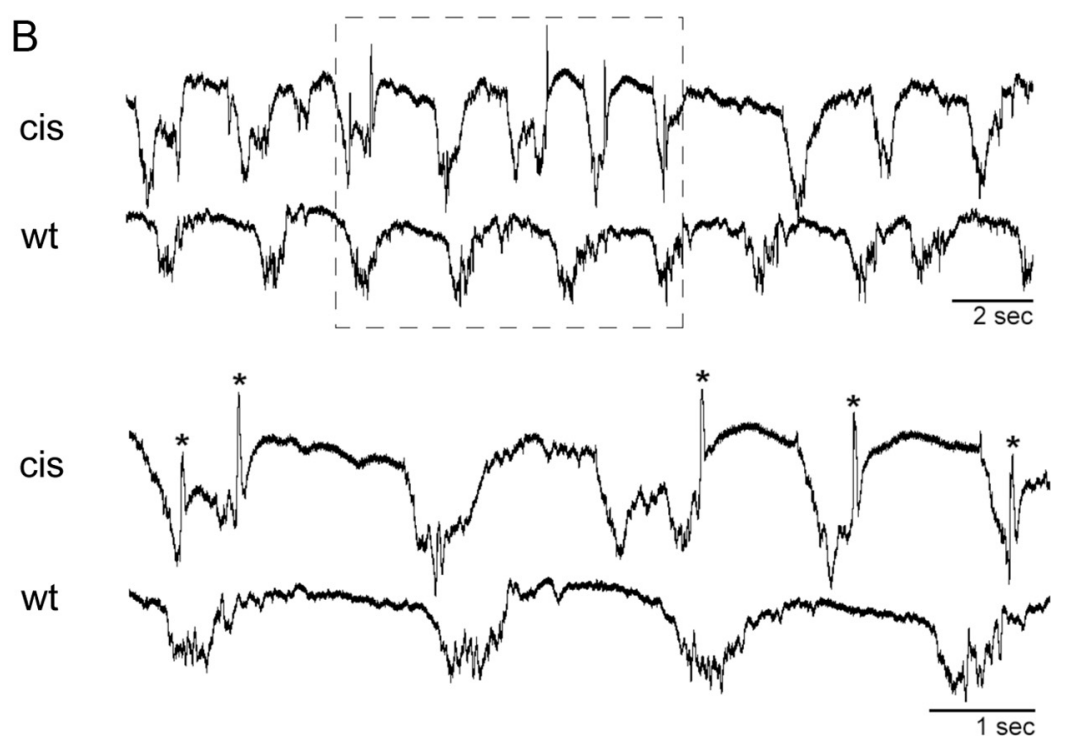

C

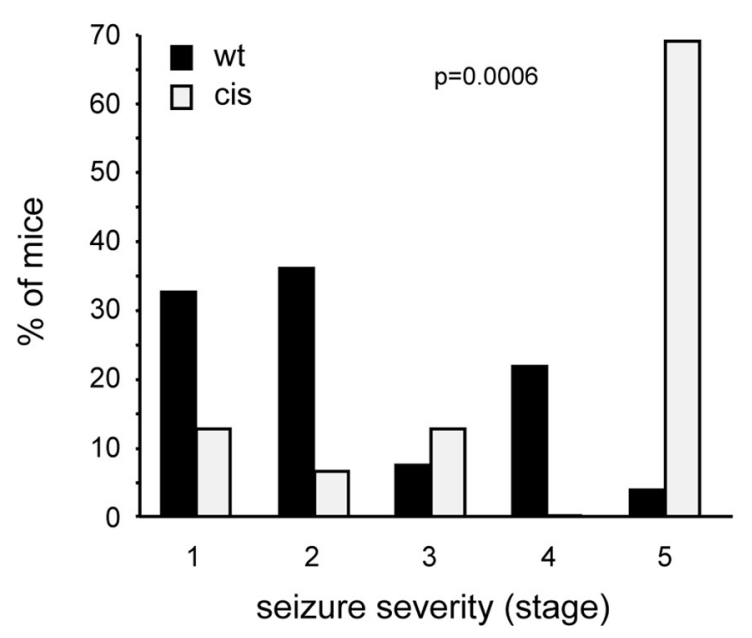

Figure 6. Decreased inhibitory activity in the cerebral cortex of cis-only mice. $A$, Loss of vGAT immunoreactivity in PV holes of cis-only mice. Scale bar, $100 \mu \mathrm{m} . \boldsymbol{B}$, EEG traces from adult cis-only and wild-type cortex. Region boxed in the top is shown enlarged below. Asterisks on the cis-only trace denote sharp negative potentials. $\boldsymbol{C}$, Induction of seizures in wild-type and cis-only mice by PTZ injection. Shown are the percentages of mice displaying different stages of seizure severity. Seizure stages are based on Lau et al. (2000). $p=0.0006$, Mann-Whitney nonparametric analysis. 


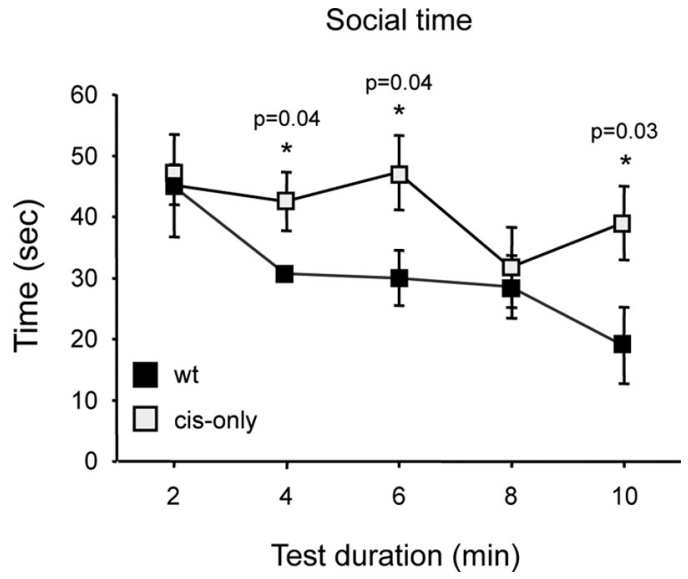

Figure 7. cis-only mice display increased social behavior. Social interaction time plotted at 2 min intervals during the test period of $10 \mathrm{~min}$.

mals, namely the frontal and visual cortices. These findings have important implications for our understanding of GDNF signaling, the diversification of GABAergic interneurons, and mechanisms of neocortical regionalization.

\section{RET-independent GDNF signaling in GABAergic interneuron development}

Our previous studies had demonstrated that mice lacking GDNF or GFR $\alpha 1$ show a reduction in the number of cortical GABAergic interneurons at birth (Pozas and Ibáñez, 2005). Since this phenotype was not reproduced in animals lacking RET, we suggested the participation of RET-independent GDNF signaling in GABAergic interneuron development. Detailed analysis of the CNS of cis-only mice has demonstrated a perfect correspondence between RET and GFR $\alpha 1$ expression and a complete absence of GFR $\alpha 1$ in cells lacking RET, without any ectopic GFR $\alpha 1$ expression (H. Enomoto, unpublished observations). Since these mice lack GFR $\alpha 1$ only in cells that do not normally express RET, processes that are controlled or regulated in a cell-autonomous manner by GDNF signaling through RET should not be affected by the cis-only mutation. In the initial analysis of these mice, no abnormalities could be observed in kidney histogenesis, enteric nervous system development or neuromuscular connectivity (Enomoto et al., 2004). Moreover, the CNS of these animals appeared grossly normal. Our present results, demonstrating profound abnormalities in cortical GABAergic neuron distribution, cortical excitability and behavior in cis-only mice, provide strong evidence for the physiological importance of RET-independent signaling in vivo. The specificity of these defects was further confirmed by their absence in conditional mutants lacking RET in the CNS, or Ret ${ }^{G \mathrm{fral} /+}$ mice, expressing an additional copy of GFR $\alpha 1$ from the Ret locus. Although the p140 isoform of NCAM can also function as a signaling receptor subunit for GDNF together with GFR $\alpha 1$ (Paratcha et al., 2003), no deficits in cortical GABAergic interneurons have been observed in the newborn cortex of mice lacking this receptor (Pozas and Ibáñez, 2005). Therefore, GFR $\alpha 1$ may contribute to GABAergic neuron development by partnering with other transmembrane molecules or through alternative mechanisms, such as ligand-induced cell adhesion (Ledda et al., 2007).

\section{PV holes and neocortical regionalization}

One of the most striking observations made in the present study is the pattern of GABAergic interneuron loss in the adult cortex of cis-only mice. Although several other mutants have also shown deficits in cortical GABAergic interneurons, cis-only mice are, to the best of our knowledge, the first to display PV holes or any such pattern of interneuron loss in the cortex. Previous studies have analyzed the effects of mutations in genes affecting large numbers, if not the majority, of GABAergic neurons or their progenitors, and found reductions in the number of interneurons across large areas of the cortex (Levitt et al., 2004; Wonders and Anderson, 2006; Liodis et al., 2007). In contrast, the deficits observed in cis-only mice were mostly restricted to PV interneurons and, among these, primarily those normally residing in the visual and frontal cortices.

The reduction of GABAergic and pan-neuronal markers observed in PV holes is consistent with cell loss, rather than abnormal differentiation, and suggests-in the absence of detectable cell death - that it has a developmental origin. This is in agreement with the overall correspondence between the predominant location of PV holes and the distribution of deficits in null and cis-only mutants at birth. Together, our observations suggest that these defects originate during prenatal cortical development. During the last week of embryonic development, GABAergic neurons that had entered the cortex by tangential migration from the ganglionic eminences disperse through the marginal, intermediate and subventricular zones before invading the cortical plate (Tanaka et al., 2006). This process goes on over several days and involves tangential migration over long distances, up to 3 $\mathrm{mm}$ in the marginal zone (Tanaka et al., 2006), well in the range of the average size of PV holes. Although GABAergic neurons have been seen to disperse in multiple directions in the developing cortex (Tanaka et al., 2006), it is currently unknown whether different interneuron subtypes have distinct dispersion routes or migratory behaviors. The predominant locations of the deficits observed in newborn and adult cis-only mutants, at the caudal and rostral poles of the cortex, suggests a role for GFR $\alpha 1$ signaling in the allocation of PV interneurons to those cortical areas. Since the caudal and frontal cortices are the regions furthest away from the ganglionic eminences, a general decrease in neuronal migration could in theory result in specific deficits in those areas. However, PV holes have not been observed in other mutants affected in general aspects of cortical interneuron migration or development, such as $u$ PAR (Powell et al., 2001), ErbB4 (Flames et al., 2004), or Lhx6 (Liodis et al., 2007) knock-out mice. We thus favor the idea that GFR $\alpha 1$ plays a more specific role in the allocation of PV interneurons to distinct cortical regions. GFR $\alpha 1$ may function in a cell-autonomous manner in a subpopulation of GABAergic precursors of the MGE, directing their differentiation and migration to specific cortical areas. Alternatively, GFR $\alpha 1$ may contribute to cortical interneuron allocation in a non-cell-autonomous manner, perhaps by regulating the interaction of interneurons with other cells or axonal pathways along their migratory routes. Because GABAergic neurons no longer express GFR $\alpha 1$ once in the cortex, deciding between these alternatives will require additional studies, including genetic fatemapping of GFR $\alpha 1$-expressing precursors and conditional ablation of GFR $\alpha 1$ in MGE cells. Regardless of the mechanisms involved, our results present the first indication of compartmentalization of a distinct GABAergic interneuron subtype in the cerebral cortex, and reveal a level of cortical regionalization that has previously remained underappreciated.

After birth, cortical interneurons undergo a protracted period of maturation during which they acquire their adult biochemical and functional properties. In the visual cortex, this stage coincides with the critical period of ocular dominance and other 
forms of plasticity, during which neural activity has the strongest influence on cortical maturation. Our observation that visual deprivation during the first 5 postnatal weeks did not affect the distribution of PV holes in the cis-only cortex is also in agreement with an earlier developmental origin for this defect, as discussed above. On the other hand, the increased size of PV holes in the visual cortex after ONT suggests that neuronal activity can influence the extent of PV interneuron loss in the affected areas. During cortical maturation, and as interneurons incorporate into functional circuits, differences in neuronal activity between cortical areas may affect interneuron distribution by recruiting cells toward the area undergoing faster activity-driven maturation. Such a process could possibly contribute to the amplification and sharpening of interneuron deficits initially established earlier in development. In agreement with our findings, an activity-driven process of this kind would not be expected to take place in identical manner in different brains, although strong correlations should be observed between hemispheres of the same brain.

\section{PV interneurons and cortical function}

The cis-only cortex displayed a major decrease in inhibitory innervation in the affected areas, abnormal EEG profiles, and markedly enhanced sensitivity to PTZ-induced seizures. All these alterations are consistent with increased cortical excitability in the cis-only brain. Changes in excitatory/inhibitory balance are believed to contribute to the pathogenesis of several cognitive disorders, including epilepsy, schizophrenia, autism, and bipolar disorder (Eyles et al., 2002; Rubenstein and Merzenich, 2003; Cossart et al., 2005). In the case of epilepsy and schizophrenia, most human studies have indicated that reduced numbers of cortical GABAergic interneurons and decreased inhibitory activity may contribute to these conditions (Levitt, 2005; Sakai et al., 2008). Interestingly, a previous study has suggested a potential linkage of Gdnf and Gfral genes to schizophrenia (Moises et al., 2002). On the other hand, the association of autism with reduced inhibitory drive is far more tenuous, mainly derived from an elevated incidence of epilepsy among a subset of autistic patients (Rubenstein and Merzenich, 2003). In fact, recent experimental evidence would appear to indicate the opposite. Mutant mice carrying a point mutation in neuroligin-3 that has been implicated in several cases of autism showed enhanced inhibitory synaptic transmission in the cerebral cortex (Tabuchi et al., 2007). Interestingly, similar to autistic patients, these animals displayed impaired social behavior. Our observations in cis-only mice showing decreased cortical inhibitory activity and enhanced social behavior are in agreement with this relationship. Together, these data suggest that, at least in mice, cortical excitability and social behavior are positively correlated.

In conclusion, the results of this study reveal the GDNF receptor GFR $\alpha 1$ as an important determinant of cortical GABAergic neuron diversification and allocation of specific interneuron subtypes to distinct cortical areas. The unusual defects of the adult cis-only cortex suggest the existence of previously unknown mechanisms of cortical regionalization which remain to be elucidated.

\section{References}

Airaksinen MS, Saarma M (2002) The GDNF family: signalling, biological functions and therapeutic value. Nat Rev Neurosci 3:383-394.

Alcantara S, de Lecea L, Del Rio JA, Ferrer I, Soriano E (1996) Transient colocalization of parvalbumin and calbindin D28k in the postnatal cerebral cortex: evidence for a phenotypic shift in developing nonpyramidal neurons. Eur J Neurosci 8:1329-1339.

Butt SJ, Fuccillo M, Nery S, Noctor S, Kriegstein A, Corbin JG, Fishell G
(2005) The temporal and spatial origins of cortical interneurons predict their physiological subtype. Neuron 48:591-604.

Corbin JG, Nery S, Fishell G (2001) Telencephalic cells take a tangent: non-radial migration in the mammalian forebrain. Nat Neurosci 4 [Suppl]:1177-1182.

Cossart R, Bernard C, Ben-Ari Y (2005) Multiple facets of GABAergic neurons and synapses: multiple fates of GABA signalling in epilepsies. Trends Neurosci 28:108-115.

Enomoto H, Hughes I, Golden J, Baloh RH, Yonemura S, Heuckeroth RO, Johnson EM Jr, Milbrandt J (2004) GFRalphal expression in cells lacking RET is dispensable for organogenesis and nerve regeneration. Neuron 44:623-636.

Estelles J, Lluch J, Rodríguez-Arias M, Aguilar MA, Miñarro J (2007) Cocaine exposure during adolescence affects anxiety in adult mice. Brain Res Bull 71:393-403.

Eyles DW, McGrath JJ, Reynolds GP (2002) Neuronal calcium-binding proteins and schizophrenia. Schizophr Res 57:27-34.

Flames N, Long JE, Garratt AN, Fischer TM, Gassmann M, Birchmeier C, Lai C, Rubenstein JL, Marín O (2004) Short- and long-range attraction of cortical GABAergic interneurons by neuregulin-1. Neuron 44:251-261.

Flames N, Pla R, Gelman DM, Rubenstein JL, Puelles L, Marín O (2007) Delineation of multiple subpallial progenitor domains by the combinatorial expression of transcriptional codes. J Neurosci 27:9682-9695.

Fogarty M, Grist M, Gelman D, Marín O, Pachnis V, Kessaris N (2007) Spatial genetic patterning of the embryonic neuroepithelium generates GABAergic interneuron diversity in the adult cortex. J Neurosci 27:10935-10946.

Kramer ER, Knott L, Su F, Dessaud E, Krull CE, Helmbacher F, Klein R (2006) Cooperation between GDNF/Ret and ephrinA/EphA4 signals for motor-axon pathway selection in the limb. Neuron 50:35-47.

Lau D, Vega-Saenz de Miera EC, Contreras D, Ozaita A, Harvey M, Chow A, Noebels JL, Paylor R, Morgan JI, Leonard CS, Rudy B (2000) Impaired fast-spiking, suppressed cortical inhibition, and increased susceptibility to seizures in mice lacking Kv3.2 $\mathrm{K}+$ channel proteins. J Neurosci 20:9071-9085.

Lavdas AA, Grigoriou M, Pachnis V, Parnavelas JG (1999) The medial ganglionic eminence gives rise to a population of early neurons in the developing cerebral cortex. J Neurosci 19:7881-7888.

Ledda F, Paratcha G, Sandoval-Guzmán T, Ibáñez CF (2007) GDNF and GFR $\alpha 1$ promote formation of neuronal synapses by ligand-induced cell adhesion. Nat Neurosci 10:293-300.

Levitt P (2005) Disruption of interneuron development. Epilepsia 46 [Suppl 7]:22-28.

Levitt P, Eagleson KL, Powell EM (2004) Regulation of neocortical interneuron development and the implications for neurodevelopmental disorders. Trends Neurosci 27:400-406.

Liodis P, Denaxa M, Grigoriou M, Akufo-Addo C, Yanagawa Y, Pachnis V (2007) Lhx6 activity is required for the normal migration and specification of cortical interneuron subtypes. J Neurosci 27:3078-3089.

Marín O, Rubenstein JL (2003) Cell migration in the forebrain. Annu Rev Neurosci 26:441-483.

Marín O, Yaron A, Bagri A, Tessier-Lavigne M, Rubenstein JL (2001) Sorting of striatal and cortical interneurons regulated by semaphorin-neuropilin interactions. Science 293:872-875.

Markram H, Toledo-Rodriguez M, Wang Y, Gupta A, Silberberg G, Wu C (2004) Interneurons of the neocortical inhibitory system. Nat Rev Neurosci 5:793-807.

Miyoshi G, Butt SJ, Takebayashi H, Fishell G (2007) Physiologically distinct temporal cohorts of cortical interneurons arise from telencephalic olig2expressing precursors. J Neurosci 27:7786-7798.

Moises HW, Zoega T, Gottesman II (2002) The glial growth factors deficiency and synaptic destabilization hypothesis of schizophrenia. BMC Psychiatry 2:8.

Paratcha G, Ledda F, Ibáñez CF (2003) The neural cell adhesion molecule NCAM is an alternative signaling receptor for GDNF family ligands. Cell 113:867-879.

Paxinos G, Franklin KBJ (2001) The mouse brain in stereotaxic coordinates, Ed 2. San Diego: Academic.

Pichel JG, Shen L, Sheng HZ, Granholm AC, Drago J, Grinberg A, Lee EJ, Huang SP, Saarma M, Hoffer BJ, Sariola H, Westphal H (1996) Defects in enteric innervation and kidney development in mice lacking gdnf. Nature 382:73-76. 
Polleux F, Whitford KL, Dijkhuizen PA, Vitalis T, Ghosh A (2002) Control of cortical interneuron migration by neurotrophins and PI3-kinase signaling. Development 129:3147-3160.

Powell EM, Mars WM, Levitt P (2001) Hepatocyte growth factor/scatter factor is a motogen for interneurons migrating from the ventral to dorsal telencephalon. Neuron 30:79-89.

Pozas E, Ibáñez CF (2005) GDNF and GFR $\alpha 1$ promote differentiation and tangential migration of cortical GABAergic neurons. Neuron 45:701-713.

Rogers DC, Fisher EM, Brown SD, Peters J, Hunter AJ, Martin JE (1997) Behavioral and functional analysis of mouse phenotype: SHIRPA, a proposed protocol for comprehensive phenotype assessment. Mamm Genome 8:711-713.

Rubenstein JL, Merzenich MM (2003) Model of autism: increased ratio of excitation/inhibition in key neural systems. Genes Brain Behav 2:255-267.

Sakai T, Oshima A, Nozaki Y, Ida I, Haga C, Akiyama H, Nakazato Y, Mikuni M (2008) Changes in density of calcium-binding-protein-immunoreactive GABAergic neurons in prefrontal cortex in schizophrenia and bipolar disorder. Neuropathology 28:143-150.
Steriade M, Nuñez A, Amzica F (1993) A novel slow ( $<1 \mathrm{~Hz}$ ) oscillation of neocortical neurons in vivo: depolarizing and hyperpolarizing components. J Neurosci 13:3252-3265.

Tabuchi K, Blundell J, Etherton MR, Hammer RE, Liu X, Powell CM, Südhof TC (2007) A neuroligin-3 mutation implicated in autism increases inhibitory synaptic transmission in mice. Science 318:71-76.

Tamamaki N, Yanagawa Y, Tomioka R, Miyazaki J, Obata K, Kaneko T (2003) Green fluorescent protein expression and colocalization with calretinin, parvalbumin, and somatostatin in the GAD67-GFP knock-in mouse. J Comp Neurol 467:60-79.

Tanaka DH, Maekawa K, Yanagawa Y, Obata K, Murakami F (2006) Multidirectional and multizonal tangential migration of GABAergic interneurons in the developing cerebral cortex. Development 133:2167-2176.

Tropea D, Kreiman G, Lyckman A, Mukherjee S, Yu H, Horng S, Sur M (2006) Gene expression changes and molecular pathways mediating activity-dependent plasticity in visual cortex. Nat Neurosci 9:660-668.

Wonders CP, Anderson SA (2006) The origin and specification of cortical interneurons. Nat Rev Neurosci 7:687-696. 